\title{
Telehealth for rural diverse populations: telebehavioral and cultural competencies, clinical outcomes and administrative approaches
}

\author{
Donald M. Hilty ${ }^{1,2}$, Melanie T. Gentry ${ }^{3}$, Alastair J. McKean ${ }^{4}$, Kirsten E. Cowan ${ }^{5}$, Russell F. Lim ${ }^{6}$, \\ Francis G. $\mathrm{Lu}^{7}$
}

${ }^{1}$ Northern California Veterans Administration Health Care System, Mather, CA 95655, USA; ${ }^{2}$ Department of Psychiatry \& Behavioral Sciences, UC Davis, Mather, CA 95655, USA; ${ }^{3}$ Geriatric Psychiatry Fellowship, ${ }^{4}$ Department of Psychiatry, Mayo Clinic College of Medicine and Science, Rochester, MN 55905, USA; ${ }^{5}$ Essentia Health and Child Psychiatrist Affiliated with Mayo Clinic Department of Psychiatry and Psychology, Hinckley, MN 55037, USA; ${ }^{6}$ Emeritus Clinical Professor of Psychiatry \& Behavioral Sciences, ${ }^{7}$ Clinical Psychiatry \& Behavioral Sciences, Emeritus Luke \& Grace Kim Professor in Cultural Psychiatry, University of California, Davis School of Medicine, Sacramento, CA 95817, USA Contributions: (I) Conception and design: DM Hilty, RF Lim, FG Lu; (II) Administrative support: DM Hilty; (III) Provision of study material or patients: None; (IV) Collection and assembly of data: DM Hilty, RF Lim, MT Gentry; (V) Data analysis and interpretation: All authors; (VI) Manuscript writing: All authors; (VII) Final approval of manuscript: All authors.

Correspondence to: Donald M. Hilty, MD, MBA. Professor and Vice-Chair, Department of Psychiatry \& Behavioral Sciences, UC Davis, 10535 Hospital Way, Mather, CA 95655, USA. Email: donh032612@gmail.com.

\begin{abstract}
Rural health care settings are challenged to provide timely and evidence-based care, particularly for culturally diverse patients with behavioral health disorders. Telepsychiatry and telebehavioral health improve access to care and leverage scarce resources. This scoping review from January 2000 - July 2019 was conducted to see if the literature had data for two related the research questions, "What are the components of culturally competent, telepsychiatric clinical care, and what approaches have clinicians and systems taken to implement and evaluate it?" The review focused on key words in four concept areas: (I) competencies; (II) telehealth in the form of telepsychiatry, telebehavioral or telemental health; (III) culture; and (IV) health. It was done in accordance with the six-stage scoping review process in PubMed/Medline and other databases. The screeners reviewed the full-text articles for final inclusion based on inclusion (mesh of the key words) and exclusion (e.g., need for only, skills abstractly discussed) criteria. From a total of 1,118 papers, the authors found 44 eligible for full text review and found 7 papers directly relevant to the concepts. Few studies specifically discuss skills and competencies of both telehealth and cultural factors. Many organizations are attending to cultural competencies and approaches to care, but there are no specific competencies that integrate telepsychiatry or telebehavioral health with culture. Existing telepsychiatric (i.e., video, social media, mobile health) and one set telebehavioral health competencies included cultural component, including use of interpreters and language matters. Administrative adjustments are suggested to promote culturally competent care by telehealth via clinical, educational, quality improvement, program/system evaluation, and other (e.g., finance and reimbursement) interventions. More structured research is needed on development, implementation and evaluation of combined competencies in rural settings.
\end{abstract}

Keywords: Culture; telebehavioral; competency; rural; health

Received: 26 August 2019; Accepted: 22 October 2019; Published: 05 April 2020.

doi: $10.21037 /$ mhealth.2019.10.04

View this article at: http://dx.doi.org/10.21037/mhealth.2019.10.04

(C) mHealth. All rights reserved. 


\section{Introduction}

The challenge to delivering culturally appropriate rural health care has been identified as a health care priority by multiple governmental and professional organizations (1-3) including the Institute of Medicine (IOM) $(4,5)$. One definition of cultural competence is "a set of congruent behaviours, attitudes and policies that come together in a system, agency or among professionals that enable that system, agency or professions to work effectively in crosscultural situations" (6). For example, the rapid growth of the Hispanic population in rural and frontier America since 2005 (7) challenges systems to add (e.g., language services, culturally competent clinicians), adjust, and further evaluate staffing and care interventions. These systems are challenged with highly acute patients, too, as rural depressed patients have three times more hospitalizations and higher suicide rates (8). While there are standardized approaches to these challenges, rural setting subcultures also vary widely $(1,7)$.

The definition of rural varies to a degree and settings' informal resources, importance of relationships, sense of trust, and degree and impact of social connectedness may facilitate health and wellness (9). Patients and clinicianssome raised there and others new to this life-may be challenged by lack of transportation, limited educational opportunities and/or poverty. These things affect helpseeking, expression of emotion and willingness to receive behavioral health care (9-13). Even if two rural communities appear to have a similar health disparity, each community is likely to have a discretely different composition of cultural, economic and geographic determinants (14).

Workforce interventions have been directed toward knowledge, attitudes, and skills (i.e., language capacity, cultural competence) $(15,16)$. Culture is multidimensional and includes race, ethnicity, spirituality, religion, sexual preference, gender identity, geography (urban, rural, global), age, socioeconomic status, education, special populations (e.g., incarcerated) and language (15); others add socioeconomics, education and other parameters to this list (1,4-5). Cultural competence training, therefore, may need to include: understanding the central role of culture in all lives and how it shapes behavior; respect and acceptance of cultural differences; learning to effectively utilize culturally adapted and culturally specific practices; and continuous development of one's awareness of personal cultural influences, prejudices or biases (17). The literature emphasizes the need for clinicians to translate knowledge and awareness into tangible behaviors that can be evaluated in vivo $(5-6,18)$.

Clinicians' increased cultural competence has been linked to increased patient satisfaction (19), treatment adherence (20) and information seeking and sharing (20). The culture of the clinician affects the interaction, as some speak the patient's language but have a different cultural background. These factors affect the development of trust versus fear of stigmatization $(1,2)$. Significant steps toward culturally competent care over the past three decades include: (I) a focus on skills in addition to knowledge for clinician training; (II) linkage of skills to patient outcomes; (III) equipping clinicians with skills to flexibly help many diverse populations rather than specialize in one population; and (IV) emphasis of team- and interdisciplinary-based (21).

To improve overall health care in rural settings, the workforce-whether in-person or via telehealthneeds cultural and technological skills $(21,22)$ (Figure 1). Administrators, staff and other team members also face obstacles related to behavioral health care and language $(9,11,12)$. Telehealth is a way to meet the behavioral health needs of rural populations, but not all clinicians have experience and skill with rural and culturally diverse populations $(21,23)$. National behavioral health organizations like the American Psychiatric Association (1969-on), the American Psychological Association (2017, 2018), the National Association of Social Workers (2015) and the American Association for Family and Marital Therapy (2004, 2015) have position statements, policies and guidelines for culturally competent care (24-28), and more recently, the use of technology in clinical care (29-33). Additionally, professional organizations and regulatory boards attend to clinical standards (i.e., professional conduct, practice and treatment guidelines, standards of care, scope of practice) rather than telecompetencies and literally clarifying how inperson care need be adjusted via telehealth $(34,35)$.

Cultural and language differences were initially believed to be more challenging for telepsychiatry compared to in-person consultations $(36,37)$, and systems must train, implement, evaluate and finance this complex care (38). Descriptive studies have shown effectiveness of telepsychiatry across many culturally diverse populations including Hispanics/Latinos, Asians, Native American (37,39-44), Eastern Europeans and other populations (e.g., deaf or hearing impaired) (40,45). Far beyond ensuring interpreter services for limited English proficiency patients, clinician must be able to reflect on medical and psychiatric illness through the full social and cultural context (46). 


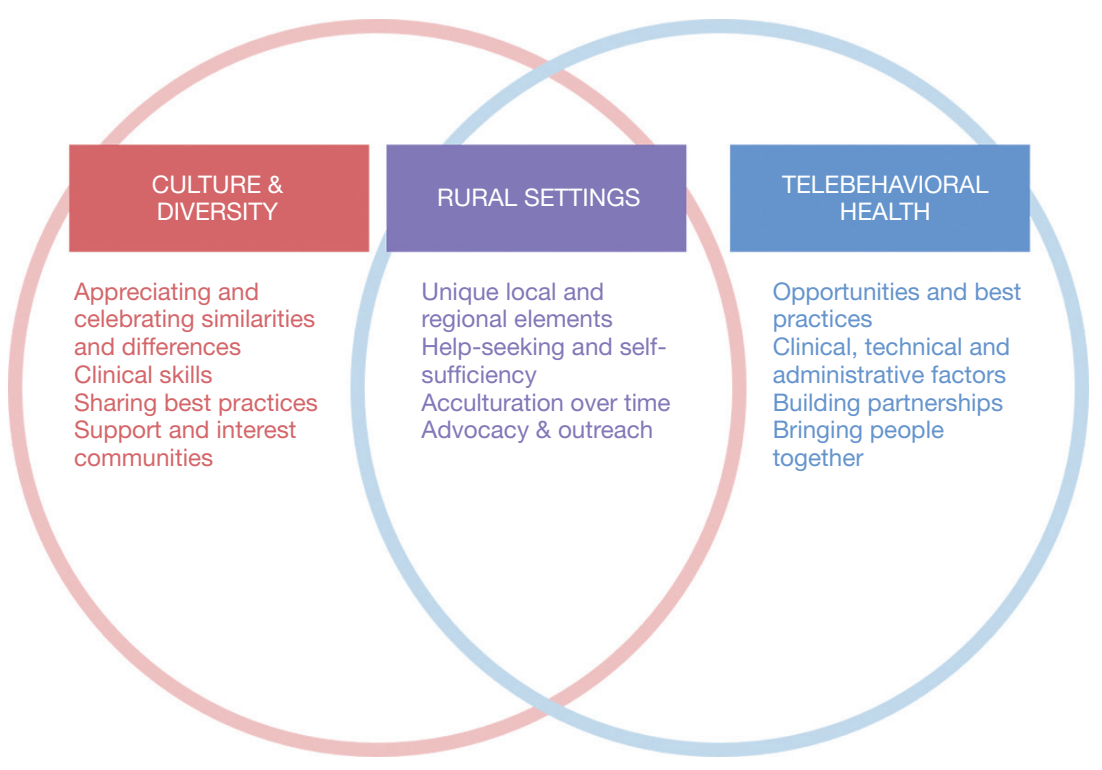

Figure 1 Integrating rural, cultural and telebehavioral health missions.

Fortunately, s spectrum of telebehavioral health care has been described (47) and telepsychiatry (i.e., video), social media, mobile health and telebehavioral telecompetencies have been developed to further this effort (48-55).

Rural primary care providers report having inadequate clinical skills to help patients with behavioral health problems (10,56). Academic health centers (AHCs), federal (e.g., veterans affairs) and statewide/county networks have begun to employ telepsychiatry to provide education, consultation and clinical care (57). "Good" delivery models like stepped, collaborative and integrated care empower and support primary care providers-rather than bypassing them-and provide structure for sustainability $(58,59)$. An approach to building these collaborations to serve rural populations emphasizes stages of needs identification, infrastructure survey, partnership organization, structural configuration and pilot implementation (36). Systems of care and their leaders employ traditional video/synchronous and asynchronous telepsychiatry-or telemental or telebehavioral health-and telephone, mobile health and other technologies.

This paper will help the reader reflect on the scoping review's research questions, "What are the components of culturally competent, telepsychiatric clinical care, and what approaches have clinicians and systems taken to implement and evaluate it?" Within that question, there are other questions including: (I) What steps have researchers, organizations and others taken to build cultural and technological components into care? (II) Have cultural and telepsychiatric competences been integrated? (III) What administrative approaches can facilitate culturally and technologically competent care, education, and evaluation in order to overcome/prevent obstacles/barriers and promote sustainability?

\section{Methods}

\section{Approach}

The literature key word search was conducted on articles published from January 2000 - July 2019 according to the original six-stage process (60) and updated modifications (61) for scoping reviews, and placed key words into concept areas (62). These reviews are typically undertaken to examine the extent, range, and nature of research in a topic area and identify gaps in knowledge rather than to examine more specific, narrow topics based on study designs of systematic reviews; both types of reviews use an approach based on concept, target population and health outcomes. Briefly, the stages in this process have been described as: (I) linking a purpose with a well-defined research question; (II) identifying relevant studies; (II) selecting studies based on an iterative process of searching the literature and refining the search strategy; (IV) charting the data and updating the form by having at least two reviewers extract information; (V) analyzing, reporting and considering the meaning of the 
findings (previously known as collating, summarizing, and reporting); and (VI) using preliminary findings to obtain consultation from stakeholders toward an aim.

The databases searched were PubMed/Medline, APA PsycNET, Cochrane, Embase, PsycINFO, Web of Science and Scopus, Embase, Science Citation Index, Social Sciences Citation Index, Telemedicine Information Exchange database, Centre for Reviews and Dissemination and The Cochrane Library Controlled Trial Registry database. The initial literature search targeted four concept areas: (I) competency(ies) (skills, behavior, cognition, cognitive, pedagogy, framework, education, training, milestones, curriculum); (II) telehealth in the form of telepsychiatry/telebehavioral or telemental health (e-, video, synchronous, asynchronous, phone, e-mail, mobile, social media); (III) culture (cultural, formulation, assessment); and (IV) health (patient, clinician, care, services, medicine, psychiatry, mental, behavioral).

\section{The research questions}

The questions that guided the review was, "What are the components of culturally competent, telepsychiatric clinical care, and what approaches have clinicians and systems taken to implement and evaluate it?" The goal was to identify behaviors (skills and competencies), make them measurable for implementation and be able to assess learning outcomes that are distinct from clinical treatment and service system outcomes.

\section{Study selection}

An iterative process involving searching the literature, refining the search strategy and reviewing articles for study inclusion was used. Two authors (DM Hilty, RF Lim) independently, in parallel, screened the search results for potentially relevant studies based on titles and abstracts. Full-text articles were reviewed for final inclusion based on the key word search. Inclusion criteria required key words from all four concept areas. Exclusion criteria included: concepts in isolation (e.g., using the word competency, but not mentioning skills); discussing skills abstractly (e.g., as part of clinical skill development); outline or listing of what clinicians need without detail (e.g., knowledge, skills and attitudes); listing behaviors that are not measureable (e.g., good engagement); and terms in combination (e.g., cognitive, Milestones, patient) without discussing competencies. When disagreements on study inclusion occurred, a third reviewer determined the outcome. Study selection would have involved post hoc inclusion and exclusion criteria, but the research question was not changed over time.

\section{Charting the data}

A data-charting form was not developed and used to extract data from each study, but notes were organized consistent with a narrative review or descriptive analytical methods by each reviewer to extract contextual or process-oriented information from each study, particularly the frameworks of telepsychiatric, telebehavioral health, social media, and mobile health competencies. The reviewers then compared and consolidated information regarding content. A descriptive analytical method was used to summarize the process and content information of discussions with experts, in an effort to chart and summarize complex concepts in a meaningful way.

The next phase was to organize meaningful results in a table, study by study, with skills outlined and parsed together incrementally. Then, consolidation could occur by the authors and expert consensus step. There were so few papers, though, that findings were reported individually, since the depth of existing research was less than expected. Indeed, there were virtually no experts outside of the authors with command of the necessary different fields (e.g., pedagogy, mobile health, medical education administration). Therefore, the reporting of results (e.g., online: http:// fp.amegroups.cn/cms/5b2dcb1db4e87a103add74474440422 7b/mhealth.2019.10.04-1.pdf) used excerpts from published framework tables; a descriptive numerical summary of results and a thematic analysis was not possible.

\section{Consultation for expert opinion}

Expert option was solicited in four ways: (I) a series of medical educator conference calls focused on teaching competencies (48-50); (II) discussion during several regional and national presentations [e.g., American Association for Directors of Psychiatry Residency Training (AADPRT)]; (III) individual discussions with educational experts (50); and (IV) two rounds of input from national behavioral health organizations (e.g., psychiatry/medicine, psychology, social work, counseling, marriage/family, psychiatric nursing, behavioral analysis) to aid in the consensus process (51-53). That process was based on an already published review of interprofessional literature (51). 
The participants included educational leaders (e.g., course/program directors, chairs, deans, a national society executive director), cultural experts, educational researchers, journal editors, and others with subject matter expertise in medicine, psychiatry, education, health services, and ethics. They represented viewpoints enriched by their leadership roles within their professional societies. Stakeholders were consulted with a purpose to validate preliminary findings, to integrate additional data related to the findings and revising the search to collect better data, if possible. Conference calls series discussed the concept areas and suggested references for review.

\section{Results}

From a total of 1,118 papers, the authors found 44 eligible for full text review and found 7 papers directly relevant to the research questions (studies in online: http:// fp.amegroups.cn/cms/5b2dcb1db4e87a103add744744042 27b/mhealth.2019.10.04-1.pdf). From papers' references, another 12 papers were found, but they were mainly foundational sources about competencies from continuing and graduate education. There were a few papers on skill development and training courses from the Office of Rural Health and Department of Veterans Affairs Employee Education System "Cultural Competence in Telehealth Clinics" (13). This aimed to increase awareness of cultural differences between patients and clinicians and ways to adapt behaviors via telehealth.

Findings were broadly categorized into the following content areas: cultural competencies and approaches to care; professional organizations' guidelines on telehealth-related care; telepsychiatric/telebehavioral health competencies with cultural components included; and an approach to integrating cultural and telebehavioral health competencies.

\section{Culturally competent care: foundations, approaches and specific training options}

Patients gain a sense from clinicians and clinics if culturally sensitive treatment is available (36). The meaning of interactions, family factors, coping styles, treatment seeking, immigration, and overall health status affect diagnosis.

It is suggested from results of this review to give patients options for professional care and communication (i.e., primary versus secondary language, in-person versus telehealth, culturally matched versus unmatched clinician, types of interpreters) $(21,38,63,64)$. "Informal" interpreters on-site like family members, nurses and others without training may miscommunicate medical complaints (65), de-emphasize information (66) and misunderstand the narrative or cultural metaphors $(11,67)$. Clinicians and staff ratings (on a Likert scale from 1 to 7 from not important to very important), though, suggest that they value use of the primary language at 5.4 over quality of care at 4.9 , access to care at 4.5 and availability of competently trained interpreter at 4.4 (11). Multicultural guidelines emphasize: exploration of identity and self-definition, as these are fluid, complex and dynamic; strength-based approaches to build resilience; culturally adaptive interventions; and advocacy across systems related to prevention, early intervention and recovery $(28,29,68)$.

In medicine, accreditation agencies in the U.S. and Canada have applied work by the Institute of Medicine toward competencies (4) (e.g., Licensing Committee for Medical Education 2015 3.3 Diversity/Pipeline Programs and Partnerships, 7.5 Social Problems and 7.6 Cultural Competence/Health Care Disparities/Personal Bias (69-73). A bio-psycho-socio-cultural (BPSC) model (74)—building upon Engel's biopsychosocial (BPS) model $(75,76)$ - has been suggested to organize behavioral health care, with expanded definition and delineation of cultural and diversity components (74). The BPSC was informed by the Outline for Cultural Formulation (OCF) and Cultural Formulation Interview (CFI) $(24,25)$. The CFI's 16 items could be shortened to a CFI-2 (i.e., two items) for medical students and a CFI-4 for residents (21). Another option is to apply questions into existing History and Physical examination templates for the history of the present illness (HPI) or Social History. Curricula in psychiatry for culture and diversity have also been implemented (15).

A systematic scoping review of health workforce cultural competency interventions found that there was significant heterogeneity in intervention strategies, measures and outcomes in terms of knowledge, skills, and attitudes/ beliefs (22). A four-dimensional conceptual framework based on educational content, pedagogical approach, structure of the intervention and participant characteristics, may be a good way to establish consensus, improve reporting and allow assessment across studies and populations (77).

\section{Behavioral health professional organizations' position statements and guidelines on telebealth or telebehavioral bealth}

A review of the telebehavioral health evidence-based 
literature across psychiatry/medicine, psychology, social work, counseling, marriage/family, behavioral analysis and other behavioral sciences found that there has been significant effort to help clinicians think about how to add/use technology and to compare in-person versus telebehavioral health (Table 1) (29-33). The subject matter of these organizations, the American Telemedicine Association (78-80) and international governmental agencies (e.g., American, British, Canadian) can be informally organized into a competency framework (Table 2), although no formal competencies have been proposed (Table 2). Professions and organizations involved with telebehavioral health could consider certification/accreditation to ensure quality care (34).

\section{Telepsychiatric and telebehavioral health competencies for clinical care and training}

A series of telepsychiatric (i.e., video) $(34,48)$, social media $(49,50)$, mobile health competencies have some emphasis on culture (53-55). These are based upon the competencybased medical education (CBME) movement, which focuses on skill development in addition to knowledge acquisition (81-84). They were developed to improve clinical care and they serve as a prototype for institutions to adjust teaching methods (e.g., curricula), faculty development and administrative policies (48). They were organized into novice/advanced beginner, competent/proficient, and expert levels; andragogical methods to teach and evaluate skills were also suggested (48).

The social media and networking competencies $(49,50)$ pose substantial challenges compared to inperson and telepsychiatric care, in that the process of care: (I) is asynchronous not synchronous, so it is cannot be "organized" or structured like traditional care; (II) creates additional boundary issues; (III) is conducted over public, private and health system sites, making the data integration and security difficult, if not impossible; and (IV) provides anonymity, with verification needed since false identities are not uncommon (53). The clinical work should be documented, since a clinician represents her/himself, the institution and the profession. Mobile health poses similar challenges and skills/competencies are needed for additional dimensions like clinical decision support, technology selection, information flow management across an e-platform and additional features (e.g., context-aware interventions and sensors with real-time feedback $(53-55,85)$.

Interprofessional telebehavioral health competencies across the specific behavioral professions have been put forward with seven domains: (I) clinical evaluation \& care, with subdomains addressing cultural competence \& diversity, documentation \& administrative procedures, social media; (II) virtual environment \& telepresence; (III) technology; (IV) legal \& regulatory issues; (V) evidencebased \& ethical practice, with a subdomain addressing social media; (VI) mobile health and apps and (VII) telepractice development addressing social media (51). These were based on input from the American Association of Marriage and Family Therapists (AAMFT), American Counseling Association (ACA), the American Psychiatric Association, American Psychological Association, the National Association for Alcoholism and Drug Abuse Counselors [NAADAC; now called, the Association for Addiction Professionals (AAP)] and the National Association of Social Workers (NASW) (29-33). The British Psychological Association and Australian Psychological Association have also contributed guidelines $(86,87)$. There are no specific competencies developed at this time for asynchronous telepsychiatry, which is a feasible, valid, reliable and costeffective in English and Spanish-speaking patients in primary care (88).

\section{The intersection of telebealth cultural competencies}

The approach to competencies has varied, with cultural components added into several domains of the ACGME framework and in the Clinical Care and Evaluation of the Coalition for Technology in Behavioral Science (CTIBS) framework. Ways in which culture affects assessment and treatment are outlined from existing competency sets (online: http://fp.amegroups.cn/cms/5b2dcb1db4e 87a103add74474404227b/mhealth.2019.10.04-1.pdf). Most commonly, culture and diversity was placed in the Interpersonal and Communication Skills ACGME domain-for video, social media and mobile health competencies. Language and use of interpreter services were placed there also. Within the ACGME competencies, culture, special populations and rural delivery are included in the Systems-based Practice and the knowledge domains. Finally, the CTIBS group included cultural issues as a component of communication under the umbrella of Telepresence, and again under the telepractice development domain.

\section{Integration of telebehavioral bealth and cultural training}

The Cultural and Linguistic Appropriate Services (CLAS) 
Table 1 The application of technology to clinical practice and training: highlights from marriage and family therapy, counseling, psychology and social work disciplines

AMERICAN COUNSELING ASSOCATION (ACA) (29) [From: ACA Code of Ethics (ACA, 2014)]

1. Knowledge and legal. Counselors who engage in the use of distance counseling, technology, and/ or social media develop knowledge and skills regarding related technical, ethical, and legal considerations (e.g., special certifications, additional course work)

2. Informed Consent and Disclosure. Clients have the freedom to choose whether to use distance counseling, social media, and/or technology within the counseling process. In addition to the usual and customary protocol of informed consent between counselor and client for face-to-face counseling, the following issues, unique to the use of distance counseling, technology, and/ or social media, are addressed in the informed consent process:
a. Informed consent and disclosure
b. Confidentiality
c. Acknowledgement of limitations
d. Security

3. Client verification

4. Distance counseling relationship
a. Benefits and limitations
b. Professional boundaries in distance counseling
c. Technology-assisted services
d. Effectiveness of services
e. Access
f. Communication differences in electronic media

5. Records and web maintenance
a. Records
b. Clients' rights
c. Electronic links
d. Multicultural and disability considerations

6. Social media
a. Virtual professional presence
b. Social media as part of informed consent
c. Client virtual presence
d. Use of public social media

MARRIAGE AND FAMILY $(30,31)$ [From: Association of Marital and Family Therapy Regulatory Board (AMFTRB) published the "Examination in Marital and Family Therapy" (AMFTRB, 2015)]

1. Maintain competence of psychology using technology: ensure their competence with both the technologies used and the potential impact of the technologies on clients/patients, supervisees, or other professionals (p. 793)

2. Ensure compliance with ethical standards: standards of care and practice are met at the outset and throughout the duration (p. 794)

3. Provide informed consent: obtain and document informed consent that specifically addresses the unique concerns related to the services and be cognizant of the applicable laws/ regulations and organizational requirements (p. 795)

4. Protect confidentiality: protect and maintain the confidentiality of the data and information relating to their client/patients and inform them of the potentially increased risk of loss of confidentiality inherent in the use of the telecommunication technologies (p. 796)

Table 1 (continued) 


\section{Table 1 (continued)}

5. Maintain security measures: protect data and information related to their clients/patients from unintended access or disclosure (p. 797)

6. Dispose data: dispose of data and information and the technologies used in a manner that facilitates protection from unauthorized access and accounts (p. 798)

7. Adjust testing practices: consider the unique issues that may arise with test instruments and assessment approaches when used via telepsychology (p. 798)

8. Comply with laws of the local jurisdiction: comply with all relevant laws and regulations of the clients/patients' jurisdictions and across international borders, if applicable (p. 799)

PSYCHOLOGY (33) [From: The American Psychological Association Guideline for the Practice of Telepsychology (American Psychological Association, 2013)]

1. Maintain competence of psychology using technology: competence with the technologies used and the potential impact of the technologies on clients/patients, supervisees, or other professionals

2. Ensure compliance with ethical standards: meet the standards of care and practice

3. Provide informed consent: obtain and document informed consent that specifically addresses the unique concerns, laws and regulations related to the services

4. Protect confidentiality: inform, protect and maintain the confidentiality of the data and information related to the use of the telecommunication technologies

5. Maintain security measures: protect data and information from unintended access or disclosure

6. Dispose data: dispose of data and information and the technologies used in a manner that facilitates protection from unauthorized access and accounts

7. Adjust testing practices: consider the unique issues that may arise with test instruments and assessment approaches when used via telepsychology

8. Comply with laws of the local jurisdiction: comply with all relevant laws and regulations of the clients/patients' jurisdictions and across international borders, as applicable

SOCIAL WORK (32)

When using technology to provide services, practitioner competence and the well-being of the client remain primary. Social workers who use technology to provide services should evaluate their ability to [From: National Association of Social Workers, Association of Social Work Boards, Council on Social Work Education, and Clinical Social Work Association, Standards for Technology in Social Work Practice, 2017]:

1. Assess the relative benefits and risks of providing social work services using technology (for example, in-person services may be necessary when clients pose a significant risk of self-harm or injurious behavior, are cognitively impaired, require sustained support by a social worker with whom they have an ongoing professional relationship, or are in crisis)

2. Reasonably ensure that electronic social work services can be kept confidential. For example, the information provided by the client should only be accessible by those who require access and that the host of the server used for electronic communication agrees to abide by the privacy policies of the social worker

3. Reasonably ensure that they maintain clear professional boundaries (for example, social workers should be mindful of boundary confusion that may result if they disclose personal information about themselves or others in an online setting to which clients have access)

4. Confirm the identity of the client to whom services are provided electronically at the onset of each contact with the client (examples include confirming a client's online consent with a telephone call; providing the client with a password, passcode, or image that is specifically for the client's use when providing consent electronically)

5. Assess individuals' familiarity and comfort with technology, access to the Internet, language translation software, and the use of technology to meet the needs of diverse populations, such as people with differing physical abilities

Table 1 (continued) 
Table 1 (continued)

The goals of the original standard were to: maintain and improve the quality of technology-related services provided by social workers, serve as a guide to social workers incorporating technology into their services, help social workers monitor and evaluate the ways technology is used in their services, and inform clients, government regulatory bodies, insurance carriers, and others about the professional standards for the use of technology in the provision of social work services [From: National Association of Social Workers developed "Standards for Technology and Social Work Practice" (NASW, 2005)]

1. Provide services via the telephone or other electronic means shall act ethically, ensure professional competence, protect clients, and uphold the values of the profession

2. Access to technology and appropriate support systems to ensure competent practice, and shall take action to ensure client access to technology

3. Select and develop appropriate online methods, skills, and techniques that are attuned to their clients' cultural, bicultural, or marginalized experiences in their environments

4. Be responsible for becoming proficient in the technological skills and tools required for competent and ethical practice and for seeking appropriate training and consultation to stay current with emerging technologies

5. Shall abide by all regulation of their professional practice with the understanding that their practice may be subject to regulation in both the jurisdiction in which the client receives services as well as the jurisdiction in which the social worker provides services

6. Shall represent themselves to the public with accuracy and make efforts to verify client identity and contact information

7. Protect client privacy when using technology in their practice and document all services, taking special safeguards to protect client information in the electronic record

8. Shall ensure high-quality practices and procedures that are legally sound and ethical to protect clients and safeguard against litigation

9. Inform and mobilize communities about policies that will benefit individuals and groups and seek to provide tools, opportunities, and information so that clients are able to advocate directly for their own interests

10. Shall strive to become and remain knowledgeable about the dynamics of online relationships, the advantages and drawbacks of non-face-to-face interactions, and the ways in which technology-based social work practice can be safely and appropriately conducted

Standards were developed through an analytical review of key legislation, regulations, contracts, and standards currently in use by federal and state agencies and other national organizations (89). The 15 standards focus on culturally competent care, including language service access and organizational supports-serving as a platform for approaches, additions and adjustments to care via telehealth.

Successful teaching of telehealth competencies requires a mixture of methods that can tailor to the needs of different learners, teachers, and settings (48). For patient care, regardless of the technologies (e.g., email, text messaging, telephone, video conferencing, apps, instructional videos, machine learning, affective computing, wearables), professionals are expected to utilize technology and therapeutically engage patients, communicate clearly and attend to boundaries and safety. Supervision with observation, role modeling and feedback are key to determine if the competencies have been achieved and to provide formative feedback $(48,52)$.

Few training programs have incorporated formal competency-based training focusing on both cultural competence and telepsychiatry. The University of Hawaii has adopted a training model for their child and adolescent fellows involving didactics and other experiences to develop culturally effective care to their rural communities utilizing telehealth (90). The Alaskan Healthcare System created telehealth and cultural competencies based on training from content experts in the regional culture that included Alaska Native Elders and University Professors (91). Rural residency programs have incorporated culturally-based competencies into their telepsychiatric education (92). This is particularly important as some First Nations/Native American and Hawaiian-Pacific Islander and Pignatiello (Canada) are often served in rural settings by telehealth than in-person care more commonly than other groups (e.g., Sick Kids program of Pignatiello, in Canada). 
Table 2 Telehealth, telepsychiatric and telebehavioral health subject matter on clinical skills and competencies for clinicians and trainees related to cultural components [note: primary organization is a modified version of the Accreditation Council of Graduate Medical Education (ACGME) framework] (85)

\begin{tabular}{|c|c|c|c|c|c|c|}
\hline Domain/competency & $\begin{array}{l}\text { Psychology } \\
\text { (Canadian } \\
\text { and U.S. } \\
\text { combined) } \\
\text { (32) }\end{array}$ & $\begin{array}{l}\text { Social/work } \\
\text { (33) }\end{array}$ & $\begin{array}{c}\text { Marriage and } \\
\text { family therapy } \\
(30,31)\end{array}$ & $\begin{array}{l}\text { Psychiatry } \\
\text { (U.S. and } \\
\text { Canadian } \\
\text { combined) } \\
(35,48)\end{array}$ & $\begin{array}{c}\text { American } \\
\text { telemedicine } \\
\text { association } \\
(81-83)\end{array}$ & $\begin{array}{c}\text { CTiBS TBH } \\
\text { Competency } \\
\text { set }(51)\end{array}$ \\
\hline \multicolumn{7}{|l|}{$\begin{array}{l}\text { Patient care (e.g., informed consent } \\
\text { and adapting care) }\end{array}$} \\
\hline \multicolumn{7}{|l|}{ Language interpreting } \\
\hline \multicolumn{7}{|l|}{$\begin{array}{l}\text { Communication, Cultural issues, } \\
\text { Language interpreting }\end{array}$} \\
\hline \multicolumn{7}{|l|}{$\begin{array}{l}\text { Culture (actual section in } \\
\text { competencies) }\end{array}$} \\
\hline \multicolumn{7}{|l|}{ Knowledge } \\
\hline \multicolumn{7}{|l|}{ Professionalism } \\
\hline \multicolumn{7}{|l|}{$\begin{array}{l}\text { Practice-based learning (e.g., } \\
\text { Quality improvement) }\end{array}$} \\
\hline \multicolumn{7}{|l|}{$\begin{array}{l}\text { Systems-based practice (e.g., } \\
\text { legal/regulatory issues) }\end{array}$} \\
\hline \multicolumn{7}{|l|}{$\begin{array}{l}\text { Technology (e.g., selection, } \\
\text { technical skills) }\end{array}$} \\
\hline \multicolumn{7}{|l|}{ Training (e.g., methods, evaluation) } \\
\hline \multicolumn{7}{|l|}{ Social media } \\
\hline
\end{tabular}

$\checkmark$, important; $\checkmark \checkmark$, discussed in-depth on importance and evaluation; $\checkmark \checkmark \checkmark$, competency set available.

\section{Physician and workflow barriers of telepsychiatry and telebehavioral health}

Health care systems are increasingly using telepsychiatry/ telebehavioral health to provide services, as rural areas of the U.S. are home to approximately $20 \%$ of the overall population, but less than $8 \%$ of the nation's physicians (e.g., psychiatrists) and other behavioral health providers (93-95). Barriers regarding physician implementation of telepsychiatry have been characterized into three categories
(I) clinical barriers; (II) workflow and technology barriers; (III) licensure, credentialing; (IV) outcome evaluation via organizational fitness, function, and leadership; (V) program/system evaluation; (VI) quality improvement; and (VII) finance and reimbursement $(12,38,58,65,96)$. Clinician barriers include concerns that they will have difficulty establishing rapport, establishing a good clinical relationship and assessing non-verbal signs of psychiatric illness (e.g., initial greeting, poor hygiene, alcohol on breath). This is best handled by technology training, sitting in on others 
Table 3 An approach to create partnerships between organizations to administratively promote culture and diversity

Overview

Example Organization (Academic) and Stakeholders: Faculty - Trainee - Department - School/Academic Health Center/University Level

Partnering Organization: Rural Clinic, Hospital and Health Network and Community (Local/Regional/National/International)

Academic organization

Faculty

Experience, attitudes and interest

Excellence in clinical care with culture and diversity

Evaluation and scholarship of educational activities

Prioritization in curricula, grand rounds, special events, retreats

Local, regional, national and international involvement and leadership

Trainees

Experience, attitudes and interest

The expectation to serve a diverse, complex and challenging population

Trainee input, feedback, partnership and leadership

Scholarship, quality improvement and health services projects

Department Administration

Central

Mission inclusion of culture and diversity

Budgetary items and alignment

Faculty-managerial-staff integration of roles

Response to critical incidents

Recruitment, promotion and retention of leaders, faculty, staff and others

Critical mass of faculty teachers and medical educators

Education and other faculty leaders

Vice-chair(s), fellowship directors, the residency training director and associate directors, clinical sites directors and the director of medical student education in psychiatry are equally participatory and well-integrated

Adherence to, and initiative/innovation beyond, national accreditation standards (e.g., ACGME, LCME)

Patient care and outreach to clinical partners

Encouragement of, expectation towards, and support of other departments and other affiliates (e.g., county, veterans affairs, community mental health, private healthcare organizations) who partner in educational, clinical and research missions

Enhancement and support of patient-centered, culturally competent care by providers

Adherence to, and initiative/innovation beyond, national accreditation standards (e.g., Joint Commission)

Facilitating clinical care via new technologies and e-services to the points-of-care needed by patients, along with use of interpreters and other professionals, to maintain a high level of care for the entire population served, regardless of race, ethnicity, language, geographic, and other potential obstacles

Table 3 (continued) 
Table 3 (continued)

\author{
School/Academic Health Center/University Level \\ Leadership, faculty and workforce \\ Faculty development (as above) \\ Education (associate deans, directors, course, and other leaders) \\ Healthcare \\ Partnering Organization (Community, Rural Network) \\ Clinic/hospital \\ Sense of ownership in mission \\ Interprofessional teamwork \\ Stepped or comparable model of care \\ Network/system
}

Communication

Shared expertise

Standardization

Registry, if feasible

Community including persons, patients, families and other interested partners

Local, state and federal organizations that facilitate access to, and treatment for, the underserved

Funding and programming directed clinical populations with diversity of languages, cultures, ages and other differences represented (e.g., threshold languages as defined by the California Cultural Competence Plan)

Faculty development, education/training, and other initiatives for baseline, incremental and critical incident-based learning and application

Recruitment, promotion and retention of leaders, faculty, staff and others

telebehavioral health clinics, and experience.

Clinical workflow and technology barriers include the additional time to plan, organize and coordinate operations for a telebehavioral health visit, which are not needed for an in-person encounter (e.g., room preparation, different location, equipment). An instrumental step is the creation of a culture in which in-person and telebehavioral health care are integrated as part of workflow. Telebehavioral health best practices include orienting patients and encouraging staff flexibility, clinician-distant site coordination and integrating in-person and tele visits. The electronic health record (EHR) may help with integration and coordination of the components of care. The use of patient questionnaires and other data (e.g., mobile health apps) may reduce interview and decision-making time and documentation.

\section{Themes in successful administrative approaches}

Successful telepsychiatric intervention models and modules for rural primary care have some common denominators, according to work in the U.S. Australia, Canada and England $(58,97)$. These models provide/obtain: (I) mutual incentive for primary care and specialty partners; (e.g. improved quality of care); (II) commitment from physicians, staff, and administration to pursue telemedicine and alternate modes of consultation; (III) systematic or multiple interventions; that meet the needs of the site; (IV) a system approach for the primary care system to monitor the flow of patients and measure outcomes; and (V) consultants who are able to bridge the differing philosophies that may be unique to rural primary care and urban academic settings and to specific cultural groups. 
A telebehavioral consultation model appears welladvised to develop skills for the primary care provider (e.g., triage, diagnosis, new or adjusted treatment plans) and since other models (e.g., managing the patient) are time intensive for the specialist $(97,98)$. Distance consultation reduces provider isolation, provides case-based learning (99) and aides decision support (100) as part of an ongoing relationship (97). Examples are: in-person and telephone doctor-to-doctor "curbside" consultations; telephone or e-mail doctor-to-doctor "curbside" consultations; case review with primary care providers; one-time cultural consultation, and distance neurocognitive assessment. These interventions have helped patients receive adequate doses of antidepressants, recover from depression $(97,101)$, accelerate service delivery (57) and avoid referral out of the community (102).

For specifics of evaluating outcomes, there are three main resources in the literature. First, ATA telemental health expert consensus that produced a lexicon for outcomes in the following areas: patient satisfaction (i.e., access, distance to service, use of), clinician satisfaction, process of care (e.g., no-shows, coordination, completion of treatment), communication (e.g., rapport), reliability/ validity (e.g., assessment, treatment $v s$. in-person), specific disorder measures (e.g., symptoms), cost (i.e., length of service, travel, hard and software) and other administrative factors (e.g., facility management, team staffing) (103). Second, a review on effectiveness systematically describes patient outcomes, models of care, and how to adjust telemental health to different populations and settings (45). Finally, high-intensity model (e.g., collaborative care) have measures to show impact at a population level (104).

\section{Discussion}

Rural health care systems must adapt significantly to provide culturally competent care via telebehavioral health (21). Principles, approaches and interventions for culturally competent care are equally important in rural and urban settings for health, generally, and behavioral health specifically. Linguistic, cultural and racial concordance have been found to facilitate patient and clinician satisfaction, as well as patient adherence to treatment (105-109). Cultural competency training for clinicians improves skills and clinical outcomes (110), but there is heterogeneity in intervention strategies and measures in health care settings, resulting in varied success with knowledge, skills, and attitudes/beliefs outcomes $(17,111)$. A conceptual framework for cultural interventions (80) is needed to assess acceptability, appropriateness, feasibility, fidelity, cost, penetration, and sustainability (112). Telehealth, by reducing distance as a barrier to treatment, has the potential to improve care if clinicians have cultural and telehealth skills.

Awareness of cultural factors has been increasing across health professions along with how the factors apply to rural settings. Instead of adding or appending curricula, competencies and policies, it is suggested to use cultural and telehealth factors to redesign clinical approaches, paradigms and models more broadly. A BPSC approach is suggested, too (77), yet some question whether there is a need to separate out social and cultural elements. There are four primary reasons to do this $(15,38,113)$ :

(I) Cultural issues may be lumped into the social history out of convenience, but that section of the interview already typically includes familial, occupational and recreational substance use;

(II) While the social history is highly emphasized, it still competes for time with presenting symptoms, past medical and behavioral health history and many other priorities in a health care visit;

(III) Clinicians can fare better by keeping culture as part of the general approach or an explanatory model for individual, family, community, societal and other events (i.e., cultural components steer helpseeking, set expectations, and influence therapeutic relationships); and

(IV) Complexity of cultural issues is not well-addressed by checklists and other approaches which may inadvertently over-simplify and stereotypein other words-unintentionally contribute to vulnerability, suffering, and missed opportunities for care.

Accordingly, it is suggested to expand the outline of skills and knowledge in (online: http://fp.amegroups.cn/cms/5b2 dcb1db4e87a103add74474404227b/mhealth.2019.10.04-1. pdf) with an ACGME framework with culture considered for each domain section.

Significant effort is needed both to translate the best practices by the IOM and other health care organizations to rural service delivery related to culture and telebehavioral health. Culture, diversity and leadership paradigms transform community practices (114). It is suggested that to reduce silos, leaders get organizational, expert and professional groups to come together (e.g., culture and technology; academic and rural; psychiatry, psychology 
and other behavioral health). Organizations aim to reduce uncertainties, improve effectiveness, and inform decisions on the goals, concerns, and perspectives of program stakeholders-and then identify program priorities and what constitutes "success" and then forge alliances. Attending to cultural and telebehavioral health issues may also have tangible and/or intangible impact on: (I) workforce recruitment and retention, (II) team and interdisciplinarybased collaboration, (III) partnerships in the community with other non-profits; (IV) adhering to and using state and federal funding streams; and (V) engagement with national leaders (e.g., American Telemedicine Association) (Table 3). Training is one way to bring both clinicians and trainees together to focus on technology and cultural competency sets; many programs have neither cultural or telepsychiatric training. Faculty/clinicians, though, need to have attitudes and skills to assess both sets, though theoretically two sets of faculty could be used. Training curricula need to set goals and align teaching and evaluation toward both sets of clinical skills.

Administrative approaches need planning, implementing, managing and evaluating programs (38). Flexible basic or advanced approaches to culturally competent care, telebehavioral health and program administration are suggested. The foundation of good program administration includes support and involvement from all levels of the organization and an interdisciplinary team who shares responsibilities and overlaps roles. If a team is changed or one is built to achieve culturally competent telebehavioral health care, core characteristics or components include, but are not limited to: leaders, clinicians, administrators, cultural consultant(s), technology (i.e., EHR computers, telemedicine), program evaluators (e.g., design, outcomes, approach, methods, analyses), scholarship/ dissemination (e.g., grant/funding proposal, reports), and/or economist/business experts (e.g., business plans, costs),. Interdisciplinary teamwork to share knowledge, skills, attitudes and experience is usually the best approach, since most systems cannot build such "dream" teams. Management of communication, change and conflict is essential.

There are limitations to this scoping review of integrated cultural and telebehavioral health. Overall, there is a thin evidence-base, aside from individual studies cited, which limits findings. Second, this is a brief overview of complex topics (e.g., culturally competent care, telebehavioral health) in order to lay a foundation toward integration between them. Third, with regard to reporting and considering the meaning of the findings, only a thematic analysis was presented, rather than a numerical analysis of the extent and nature of studies. A summary of results would be in order, but when a scoping review is done when there is "insufficient evidence," that is not always possible (115). Fourth, broader input for consensus across organizations could have been helpful, and in working with representatives, a qualitative, small group interview approach with experts using via a semi-structured guide could have asked participants to identify models for care (regardless of whether they were published) and to specify key model components (116). Finally, while there is established literature on behavioral health and ethnic groups, generalizing approaches and findings across diverse groups is not advised, without additional research evidence.

\section{Conclusions}

Rural healthcare systems are challenged to provide timely, evidence-based care, particularly for culturally diverse patients with behavioral disorders. Telepsychiatry and/ or telebehavioral health improve access to care and telecompetency sets are beginning to integrate cultural and telehealth skills. Program and system administrators can support culturally competent care by telehealth via clinical, educational and quality improvement training and evaluation. It is particularly important to build, train and sustain capacity of workforce. Interprofessional, interdisciplinary and inter-organizational team approaches are suggested across local, regional and (inter)national communities. More structured research is needed to develop, implement and evaluate combined competencies in rural settings.

\section{Acknowledgments}

Coalition for Technology in Behavioral Science (CTIBS); UC Davis School of Medicine and Department of Psychiatry \& Behavioral Sciences; Veteran Affairs Northern California Health Care System and Mental Health Service. Funding: None.

\section{Footnote}

Conflicts of Interest: The authors have no conflicts of interest to declare.

Ethical Statement: The authors are accountable for all 
aspects of the work in ensuring that questions related to the accuracy or integrity of any part of the work are appropriately investigated and resolved.

Open Access Statement: This is an Open Access article distributed in accordance with the Creative Commons Attribution-NonCommercial-NoDerivs 4.0 International License (CC BY-NC-ND 4.0), which permits the noncommercial replication and distribution of the article with the strict proviso that no changes or edits are made and the original work is properly cited (including links to both the formal publication through the relevant DOI and the license). See: https://creativecommons.org/licenses/by-nc$\mathrm{nd} / 4.0 /$.

\section{References}

1. Office of the Surgeon General Center for Mental Health Services and National Institute of Mental Health. Mental Health: Culture, Race, and Ethnicity: A Supplement to Mental Health: A Report of the Surgeon General 2009, Available online: http://www.ncbi.nlm.nih.gov/books/ NBK44242/.

2. Berger JT. Culture and ethnicity in clinical care. Arch Intern Med 1998;158:2085-90.

3. Department of Justice. Title VI of the Civil Rights Act of 1964, Available online: https://www.justice.gov/crt/fcs/ TitleVI.

4. Smedley B, Stith A, Nelson A, (eds). Unequal Treatment: Confronting Racial and Ethnic Disparities in Health Care. National Academies Press, Washington, DC, 2003.

5. Smedley BD, Butler AS, Bristow LR (eds). In the Nation's Compelling Interest: Ensuring Diversity in the Health Care Workforce. Institute of Medicine (IOM) of The National Academies. Washington, D.C.: The National Academies Press 2004, Available online: http://www.nap. edu/books/030909125X/html/.

6. Cross TL, Bazron B, Dennis K, et al. Towards a culturally competent system of care: a monograph on effective services for minority children who are severely emotionally disturbed. Georgetown University Child Development Center, Washington, DC, 1989, Available online: https://www.ncjrs.gov/App/Publications/abstract. aspx?ID=124939.

7. U.S. Department of Agriculture. What is Rural? 2006, Available online: http://ric.nal.usda.gov/what-is-rural/.

8. Rost K, Zhang M, Fortney J, et al. Rural-urban differences in depression treatment and suicidality. Med Care
1998;36:1098-107.

9. Holmes C, Levy M. Rural Culture Competency in Health Care White Paper. 2015. Available online: https:// reachhealth.org/wp-content/uploads/2016/12/REACHRCC-White-Paper-Final.pdf

10. Geller JM, Muus KJ. The role of rural primary care physicians in the provision of mental health services. J Wash Acad Sci 2000;86:131-42.

11. Hilty DM, Lim RF, Nasatir-Hilty SE, et al. Planning for telepsychiatric consultation: A needs assessment for cultural and language services at rural sites in California. J Rur Ment Health 2015;39:153-61.

12. Brooks E, Manson SM, Bair B, et al. The diffusion of telehealth in rural American Indian communities: a retrospective survey of key stakeholders. Telemed J E Health 2012;18:60-6.

13. Office of Rural Health and Department of Veterans Affairs Employee Education System (Veterans Health Administration Office of Care Coordination Services. Cultural Competence in Telehealth Clinics 2017, Available online: https://www.ruralhealth.va.gov/docs/culturalcompetence-in-telehealth-clinics.pdf.

14. Thomas TL, DiClemente R, Snell S. Overcoming the triad of rural health disparities: How local culture, lack of economic opportunity, and geographic location instigate health disparities. Health Educ J 2014;73:285-94.

15. Lim RF, Lu F. Culture and psychiatric education. Acad Psychiatry 2008;32:269-71.

16. Betancourt JR, Green AR, Carrillo JE, et al. Defining cultural competence: a practical framework for addressing racial/ethnic disparities in health and health care. Public Health Rep 2003;118:293-302.

17. Purnell LD. Transcultural Health Care: A Culturally Competent Approach. Vol. 4. FA Davis Company, Philadelphia, 2012.

18. Napier AD, Ancarno C, Butler B, et al. Culture and health. Lancet 2014;384:1607-39.

19. Paez KA, Allen JK, Beach MC, et al. Physician cultural competence and patient ratings of the patient-physician relationship. J Gen Intern Med 2009;24:495-8.

20. Roncoroni J, Tucker CM, Wall W. Patient perceived cultural sensitivity of clinic environment and its association with patient satisfaction with care and treatment adherence. Am J Lifestyle Med 2014;8:421-9.

21. Hilty DM, Evangelatos G, Valasquez A, et al. Telebehavioral health for rural culturally diverse populations: Approaches for clinical services, competencies and administration. J Tech Behav Sci 2018;3:206-20. 
22. Jongen C, McCalman J, Bainbridge R. Health workforce cultural competency interventions: a systematic scoping review. BMC Health Serv Res 2018;18:232.

23. Yellowlees P. Marks S, Hilty D, et al. Using e-health to enable culturally appropriate mental healthcare in rural areas. Telemedicine and e-Health 2008;14:486-92.

24. American Psychiatric Association. Cultural Formulation Interview. (2013). Patient Version. Website: http:// www.dsm5.org/Pages/Feedback-Form.aspx, and Informant Version 2013, Available online: http:// www.multiculturalmentalhealth.ca/wp-content/ uploads/2013/10/2013_DSM5_CFI_InformantVersion.pdf.

25. American Psychiatric Association. Cultural Formulation Interview Supplementary Modules 2013, Available online: http://www.multiculturalmentalhealth.ca/wp-content/ uploads/2013/10/2013_CFI_supplementarymodules.pdf.

26. American Psychological Association. Guidelines for Providers of Psychological Services to Ethnic, Linguistic, and Culturally Diverse Populations 2017, Available online: http://www.apa.org/pi/oema/resources/policy/providerguidelines.aspx.

27. National Association of Social Workers. Standards and Indicators of Cultural Competency in Social Work Practice 2015, Available online: https://www.socialworkers. org/LinkClick.aspx? fileticket=PonPTDEBrn4\%3D\&port alid $=0$

28. American Counseling Association. Code of Ethics 2014, Available online: https://www.counseling.org/resources/ aca-code-of-ethics.pdf.

29. American Association for Family and Marital Therapy. Core Competencies 2004, Available online: https://www.aamft. org/imis15/Documents/MFT_Core_Competencie.pdf.

30. American Association for Family and Marital Therapy. Code of Ethics 2015, Available online: http://www.aamft. org/iMIS15/AAMFT/Content/Legal_Ethics/Code_of_ Ethics.aspx.

31. American Psychological Association. Guideline for the Practice of Telepsychology 2013, Available online: http:// www.apapracticecentral.org/ce/guidelines/telepsychologyguidelines.pdf.

32. National Association of Social Workers, Association of Social Work Boards, Council on Social Work Education, and Clinical Social Work Association, 2017. Standards for Technology in Social Work Practice. Available online: https://www.naswpress.org/publications/standards/ technology.html.

33. American Association for Marriage and Family Therapy. Standard VI Technology-Assisted Professional Services,
2015, Available online: https://www.aamft.org/iMIS15/ AAMFT/Content/legal_ethics/code_of_ethics.aspx.

34. Hilty DM, Maheu M, Drude K, et al. Telebehavioral health, telemental health, e-therapy and e-health competencies: the need for an interdisciplinary framework. J Tech Behav Sci 2017;2:171-89.

35. Hilty DM, Maheu M, Drude K, et al. The need to implement and evaluate telehealth competency frameworks to ensure quality care across behavioral health professions. Acad Psychiatry 2018;42:818-24.

36. Shore JH, Manson SM. A developmental model for rural telepsychiatry. Psychiatr Serv 2005;56:976-80.

37. Shore JH, Savin DM, Novins D, et al. Cultural aspects of telepsychiatry. J Telemed Telecare 2006;12:116-21.

38. Hilty DM, Feliberti J, Evangelatos G, et al. Competent cultural telebehavioral healthcare to diverse populations: Administration, evaluation, and financing. J Tech Behav Sci 2018;4:186-200.

39. Chong J, Moreno F. Feasibility and acceptability of clinicbased telepsychiatry for low-income Hispanic primary care patients. Telemed J E Health 2012;18:297-304.

40. Moreno FA, Chong J, Dumbauld J, et al. Use of standard webcam and Internet equipment for telepsychiatry treatment of depression among underserved Hispanics. Psychiatr Serv 2012;63:1213-7.

41. Nieves JE, Stack KM. Hispanics and telepsychiatry. Psychiatr Serv 2007;58:877-8.

42. Ye J, Shim R, Lukaszewski T, et al. Telepsychiatry services for Korean immigrants. Telemed J E Health 2012;18:797-802.

43. Weiner MF, Rossetti HC, Harrah K. Videoconference diagnosis and management of Choctaw Indian dementia patients. Alzheimers Dement 2011;7:562-6.

44. Mucic D. Transcultural telepsychiatry and its impact on patient satisfaction. J Telemed Telecare 2010;16:237-42.

45. Hilty DM, Ferrer D, Johnston B, et al. The effectiveness of telemental health: A 2013 review. Telemed J E Health 2013;19:444-54.

46. Kim G, Aguado Loi CX, Chiriboga DA, et al. Limited English proficiency as a barrier to mental health service use: A study of Latino and Asian immigrants with psychiatric disorders. J Psychiatr Res 2011;45:104-10.

47. Hilty DM, Chan S, Torous J, et al. New frontiers in healthcare and technology: Internet- and web-based mental options emerge to complement in-person and telepsychiatric care options. J Health Med Inform 2015;6:1-14.

48. Hilty DM, Crawford A, Teshima J, et al. A framework for 
telepsychiatric training and e-health: Competency-based education, evaluation and implications. Int Rev Psychiatry 2015;27:569-92.

49. Hilty DM, Zalpuri I, Stubbe D, et al. Social media/ networking as part of e-behavioral health and psychiatric education: Competencies, teaching methods, and implications. J Tech Behav Sci 2018;3:268-93.

50. Zalpuri I, Liu H, Stubbe D, et al. A competency-based framework for social media for trainees, faculty and others. Acad Psychiatr 2018;42:808-17.

51. Maheu M, Drude K, Hertlein K, et al. An interdisciplinary framework for telebehavioral health competencies. J Tech Behav Sci 2018;3:108-40; correction 3:107.

52. Maheu MM, Drude K, Hertlein K, et al. A framework for interprofessional telebehavioral health competencies: Implementation and challenges moving forward. Acad Psychiatry 2018;42:825-33.

53. Hilty DM, Chan S, Torous J, et al. A telehealth framework for mobile health, smartphones and apps: competencies, training and faculty development. J Tech Behav Sci 2019;4:106-23.

54. Hilty DM, Chan S, Torous J, et al. A competency-based framework for psych/behavioral health apps for trainees, faculty, programs and health systems. Psych Clin N Amer 2019;42:513-34.

55. Hilty DM, Chan S, Torous J, et al. A framework for competencies for the use of mobile technologies in psychiatry and medicine. JMIR Uhealth and Mobile Health 2019; In Press.

56. Geller JM. Rural primary care providers' perceptions of their roles in the provision of mental health services: Voices from the plains. J Rural Health 1999;15:326-34.

57. Hilty D, Yellowlees PM, Parish MB, et al. Telepsychiatry: Effective, evidence-based and at a tipping point in healthcare delivery. Psychiatr Clin North Am 2015;38;559-92.

58. Hilty DM, Rabinowitz TR, McCarron RM, et al. Telepsychiatry and e-mental health models leverage stepped, collaborative, and integrated services to primary care. Psychosomatics 2018;59:227-50.

59. Hilty DM, Sunderji N, Suo S, et al. Telepsychiatry/ telebehavioral health and integrated care: evidence-base, best practice models and competencies. Int Rev Psychiatry 2019;1:1-18.

60. Arskey H, O'Malley L. Scoping studies: towards a methodological framework. Int J Soc Res Meth 2005;8:19-32.

61. Levac D, Colquhoun H, O'Brien KK. Scoping studies: advancing the methodology. Implement Sci 2010;5:69.

62. Platt R, Spencer A, Burkey M, et al. What's known about implementing co-located pediatric integrated care: a scoping review. In Rev Psychiatr 2018;30:242-71.

63. Yellowlees PM, Odor A., Iosif AM, et al. Transcultural psychiatry made simple: Asynchronous telepsychiatry as an approach to providing culturally relevant care. Telemed J E Health 2013;19:259-64.

64. Beach MC, Price EG, Gary T, et al. Cultural competency: A systematic review of health care provider educational interventions. Med Care 2005;43:356-73.

65. Brooks TR. Pitfalls in communication with Hispanic and African-American patients: Do translators help or harm? J Natl Med Assoc 1992;84:941-7.

66. Brua C. Role-blurring and ethical grey zones associated with lay interpreters: Three case studies. Commun Med 2008;5:73-9.

67. Elderkin-Thompson V, Silver RC, Waitzkin H. When nurses double as interpreters: A study of Spanish-speaking patients in a US primary care setting. Soc Sci Med 2001;52:1343-58.

68. APA adopts new multicultural guidelines. Monit Psychol 2018. Available online: https://www.apa.org/ monitor/2018/01/multicultural-guidelines

69. Liaison Committee on Medical Education Competencies Revision 2015, Available online: https://www.lcme.org/ publications/2015-16-functions-and-structure-withappendix.pdf.

70. American Association of Medical Colleges, Core Medical Student Competencies 2015, Available online: https:// www.aamc.org/.

71. Committee on the Accreditation of Canadian Medical Schools 2017, Available online: https://afmc.ca/ accreditation/committee-accreditation-canadian-medicalschools-cacms.

72. Lim RF, Luo J, Suo S, et al. Diversity initiatives in academic psychiatry: Applying cultural competence. Acad Psychiatry 2008;32:283-90.

73. Loue $\mathrm{S}$, Wilson-Delfosse A, Limbach K. Identifying gaps in the cultural competence/sensitivity components of an undergraduate medical school curriculum: A Needs Assessment. J Immigr Minor Health 2015;17:1412-9.

74. Hilty DM. Advancing science, clinical care and education: Shall we update Engel's Biopsychosocial Model to a BioPsycho-Socio-Cultural Model? Psychol Cogn Sci Open J 2015;1:e1-6.

75. Engel GL. The clinical application of the biopsychosocial model. Am J Psychiatry 1980;137:535-44. 
76. Engel GL. The need for a new medical model: A challenge for biomedicine. Science 1977;196:129-36.

77. Horvat L, Horey D, Romios P, et al. Cultural competence education for health professionals. Cochrane Database Syst Rev 2014;(5):CD009405.

78. American Telemedicine Association, 2009. Practice Guidelines for Videoconferencing-Based Telemental Health. Available online: http://www.americantelemed. org/docs/default-source/standards/practice-guidelines-forvideoconferencing-based-telemental-health.pdf? sfvrsn $=6$.

79. American Telemedicine Association, 2013. Practice Guidelines for Video-based Online Mental Health Services. Available online: http://www.americantelemed. org/docs/default-source/standards/practice-guidelines-forvideo-based-online-mental-health-services.pdf? $\mathrm{sfvrsn}=6$.

80. American Telemedicine Association, 2017. Practice Guidelines for Telemental Health with Children and Adolescents. Available online: https://higherlogicdownload.s3.amazonaws.com/ AMERICANTELEMED/618da447-dee1-4ee1b941-c5bf3db5669a/UploadedImages/Practice\%20 Guideline\%20Covers/NEW_ATA\%20Children\%20 \&\%20Adolescents\%20Guidelines.pdf.

81. Frank JR. The CanMEDS 2005 Physician Competency Framework 2005, Available online: http://rcpsc.medical. org/canmeds/CanMEDS2005/CanMEDS2005_e.pdf.

82. Accreditation Council on Graduate Medical Education. (2013). Common Program Requirements. Available online: https://www.acgme.org/acgmeweb/Portals/0/PFAssets/ ProgramRequirements/CPRs2013.pdf.

83. American Psychiatric Association Council on Medical Education and Lifelong Learning. (2014). Training Psychiatrists for Integrated Behavioral Health Care (Official Actions). Arlington, VA: American Psychiatric Association 2014, Available online: http://www.psychiatry. org/File\%20Library/Advocacy\%20and\%20Newsroom/ Press\%20Releases/2015\%20Releases/CMELL_ ICReport_Public_03022015.pdf.

84. Royal College of Physicians and Surgeons, CanMEDS Framework 2005, Available online: http://www. royalcollege.ca/portal/page/portal/rc/canmeds/ framework/.

85. Kumari P, Mathew L, Syal P. Increasing trend of wearables and multimodal interface for human activity monitoring: A review. Biosens Bioelectron 2017;90:298-307.

86. British Psychological Society. The Provision of Psychological Services via the Internet and Other Non-direct Means 2009, Available online: http://The_
provision_of_psychological_services_via_the_Internet.

87. Australian Psychological Society. Guidelines for Providing Psychological Services and Products Using the Internet and Telecommunications Technology 2011, Available online: https://aaswsocialmedia.wikispaces.com/file/view/ EG-Internet.pdf.

88. Yellowlees PM, Odor A, Burke MM, et al. A feasibility study of asynchronous telepsychiatry for psychiatric consultations. Psychiatr Serv 2010;61:838-40.

89. U.S. Department of Health and Human Services. (2018). National Standards for Culturally and Linguistically Appropriate Services (CLAS) in Health and Health Care. Available online: https://www.thinkculturalhealth.hhs. gov/clas.

90. Chung RC, Bemak F. The relationship of culture and empathy in cross-cultural counseling. J Counsel Devel 2002;80:154-9.

91. Hiratsuka V, Delafield R, Starks H, et al. Patient and provider perspectives on using telemedicine for chronic disease management among Native Hawaiian and Alaska Native people. Int J Circumpolar Health 2013;72:21401. doi:10.3402/ijch.v72i0.21401

92. Gifford V, Niles B, Rivkin I, et al. Continuing education training focused on the development of behavioral telehealth competencies in behavioral healthcare providers. Rural Remote Health 2012;12:2108.

93. U.S. Department of Health and Human Services, Health Resources Services Administration, author. Office of Rural Health Policy fact sheet 2009, Available online: http:// www.hrsa.gov/about/pdf/orhp.pdf.

94. Lauckner C, Whitten P. The state and sustainability of telepsychiatry programs. J Behav Health Serv Res 2016;43:305-18.

95. Cowan K, Gentry M, McKean A, et al. Barriers to use of telepsychiatry: clinicians as gatekeepers. Mayo Clin Proceed, In Press

96. Institute for Healthcare Improvement. Quality Improvement Essentials Toolkit 2018, Available online: http://www.ihi.org/resources/Pages/Tools/QualityImprovement-Essentials-Toolkit.aspx.

97. Hilty DM, Nesbitt T, Kuenneth TS, et al. Rural versus suburban primary care needs, utilization, and satisfaction with telepsychiatric consultation. J Rural Health 2007;23:163-5.

98. Katon W, Von Korff M, Lin E, et al. Collaborative management to achieve treatment guidelines impact on depression in primary care. JAMA 1995;273:1026-31.

99. Greenberg WE, Paulsen RH. Moving into the 
neighborhood: preparing residents to participate in a primary care environment. Harv Rev Psychiatry 1996;4:107-9.

100.Armstrong IJ, Haston WS. Medical decision support for remote general practitioners using telemedicine. J Telemed Telecare 1997;3:27-34.

101.Simon GE. Randomized trial of monitoring, feedback, and management of care by telephone to improve treatment of depression in primary care. Brit Med J 2000;320:550-4.

102. Marcin JP, Nesbitt TS, Struve S, et al. Financial benefits of a pediatric intensive care unit-based telemedicine program to a rural adult intensive care unit: Impact of keeping acutely ill and injured children in their local community. Telemed J E Health 2004;10 Suppl 2:S-1-5.

103. Shore JH, Mishkind MC, Bernard J, et al. A lexicon of assessment and outcome measures for telemental health. Telemed J E Health 2014;20:282-92.

104. Fortney JC, Pyne JT, Turner E, et al. Telemedicine integration of mental health into rural primary care settings. Int Rev Psychiatry 2015;27:525-39.

105. Freeman GK, Rai H, Walker JJ, et al. Non-English speakers consulting with the GP in their own language: a cross-sectional survey. Br J Gen Pract 2002;52:36-8.

106.Pöchhacker F. Language barriers in Vienna hospitals. Ethn Health 2000;5:113-9.

107. Saha S, Komaromy M, Koepsell TD, et al. Patientphysician racial concordance and the perceived quality and use of health care. Arch Intern Med 1999;159:997.

108. Bowen S. Language Barriers in Access to Health Care.

doi: $10.21037 /$ mhealth.2019.10.04

Cite this article as: Hilty DM, Gentry MT, McKean AJ, Cowan KE, Lim RF, Lu FG. Telehealth for rural diverse populations: telebehavioral and cultural competencies, clinical outcomes and administrative approaches. mHealth 2020;6:20.
Health Canada, Ottawa, Ontario, Canada, 2001.

109.Pérez-Stable EJ. Language access and Latino health care disparities. Med Care 2007;45:1009-11.

110. Ton H, Koike A, Hales RE, et al. A qualitative needs assessment for development of a cultural consultation service. Transcult Psychiatry 2005;42:491-504.

111.Jongen C, McCalman J, Bainbridge R, et al. Cultural competence in health: A review of the evidence. Springer Nature, Singapore, 2018.

112.Proctor E, Silmere H, Raghavan R, et al. Outcomes for implementation research: conceptual distinctions, measurement challenges, and research agenda. Adm Policy Ment Health 2011;38:65-76.

113. Farmer J, Bourke L, Taylor J, et al. Culture and rural health. Aust J Rural Health 2012;20:243-7.

114. Shapiro ML, Miller J, White K. Community transformation through culturally competent nursing leadership: application of theory of culture care diversity and universality and tri-dimensional leader effectiveness model. J Transcult Nurs 2006;17:113-8.

115. Monash University. Clinical guideline for the diagnosis and management of work-related mental health conditions in general practice: GP summary 2019, Available online: https://www.monash.edu/_data/assets/pdf_ file/0010/1696681/GP-Summary-of-the-Work-RelatedMental-Health-Clinical-Guideline_Digital.pdf.

116. Korthuis PT, McCarty D, Weimer M, et al. Primary carebased models for the treatment of opioid use disorder: A scoping review. Ann Intern Med 2017;166:268-78. 